\title{
Development Strategies of Movie and Television Tourism in Yunnan Based on Industrial Integration
}

\author{
Yufei Hu \\ Oxbridge College, Kunming University of Science and Technology, Kunming, Yunnan, 650000, China
}

Keywords: industry integration; Yunnan; movie and television tourism; development strategy

\begin{abstract}
The development of movie and television tourism has increasingly reflected the characteristic of industrial integration, which makes it important to study movie and television tourism from the perspective of industrial integration. Movie and television tourism is not a simple combination of movie and television with tourism. The industrial integration of the two can promote the development of movie and television tourism in depth and breadth. Movie and television tourism is relatively independent, and it is very important to explore the development strategy of movie and television tourism. Based on the author's learning and practical experience, this paper first analyzed the connotation of movie and television tourism, and then discussed the industrial integration characteristics of movie and television tourism. The paper finally proposed the development strategy of movie and television tourism in Yunnan based on the theory of industrial integration.
\end{abstract}

\section{Introduction}

The development of movie and television tourism has increasingly reflected the characteristics of industrial integration, which makes it important to study movie and television tourism from the perspective of industrial integration. From the perspective of industrial integration, the movie and television tourism development model can be divided into six categories: movie and television shooting location tourism, movie and television story tourism, movie and television culture tourism, movie and television production base tourism, movie and television theme park tourism, and movie and television festival tourism, of which the first three categories can be further summarized as movie and television communication tourism, and the latter three categories can provide content for movie and television tourism. The inherent communication function of movie and television is a foundation of the former. The non-boundary of tourism attraction is the basis of the latter. The common foundation of the two is the cultural industry essence of movie and television industry, and tourism industry. Technological innovation is the main driving force for industrial integration in the field of information technology, while the market integration caused by demand is the fundamental driving force for the development of movie and television tourism. As the field of information technology, enterprises are also the main body of the integration of movie and television industry and tourism industry.

\section{The Connotation of Movie and Television Tourism}

Movie and television tourism, called "Movie Induced Tourism" in the West, is considered to be a tourism activity that promotes tourists to visit these tourist sites and attractions because of the appearance of tourism destinations on screens, video tapes and screens (Definition 1). This definition emphasizes the role of movie and television transmission, which makes the movie and television location more popular. However, the reason why he movie and television base is attractive is not the spread and publicity of the movie and television works, but is the opportunity to capture the experience and see the stars in the movie and television base where the movie and television works are shot. Therefore, the definition proposed by Liu Binyi has been more cited: movie and television tourism is a tourism activity that involves in the whole process of movie and television, and the affairs related to movie and television (Definition 2). The mode of movie and 
television tourism industry studied in this paper is mainly for the tourist destination in Definition 1 , so the first definition is adopted here.

\section{Industrial Integration Characteristics of Movie and Television Tourism}

\subsection{The transition from passiveness to positiveness.}

The role of movie and television transmission is innate. In the 1980s, China's movie and television industry started. Although there were few movie and television works in this period, it has already reflected the powerful communication role of movie and television works. At that time, the movie "Romance on Lushan Mountain" made Lushan Scenic Area the most famous tourist attraction in China. The popular TV play "Journey to the West" produced in 1986 has a subtle influence on Huangguoshu Waterfall in Guizhou and Jiuzhaigou Valley in Sichuan for a long time. With the prosperity and development of the movie and television industry, the intangible transmission of movie and television works to tourist destinations has increasingly attracted the attention of all local governments who actively use these works or cooperate with movie and television producers to promote tourism destinations. For example, Zhangjiajie used the "Avatar" released in 2010 to change the name of Nantianyizhu in the Wulingyuan Scenic Area to the Halleluja Mountain in "Avatar", which caused strong concern and heated discussion in China. Some people think that this is the marketing means of the government and scenic spots in the post-movie economy and market economy. The cooperation between the Publicity Department of Hangzhou Municipal Committee and Feng Xiaogang's "If You Are the One" was to present the Xixi Wetland in Hangzhou to the people of the country, which enhances the city image of Hangzhou

\subsection{The transition from late publicity with the works to the early recommendation.}

In the early days of movie and television tourism development, the tourism destination used the influence of the movie and television works to promote the destination when the works are broadcast. For example, the Lushan Mountain Scenic Area broadcasts the "Romance on Lushan Mountain" for many times and includes it into one of the contents to visit Lushan Mountain. People go to Huangguoshu Waterfall and Jiuzhaigou Valley to experience the scene of "Journey to the West" produced in 1986, which adds fun to the tourists. The scene selection of the movie and television works at that time only considered the plot itself. Today, many tourist destinations actively contact and cooperate with the photographers, and involve in the location selection and script creation of the movie and television works. That is to say, in the early stage of movie shooting, plans have been made to promote the destination with the movie and television. For example, in 2003, the TV series "Time After Time" was created and made after Huang Lei was inspired in Wuzhen Village. The important sights and features of Wuzhen Village were infiltrated throughout the play. If "Time After Time" is Huang Lei's unintentional purpose, then "If You Are the One 2" is the sincere cooperation between Beijing Tourism Bureau and the movie producer. Before the shooting, Beijing Tourism Bureau consulted with Huayi Brothers Media Co., Ltd. and became its chief partner. They successfully added a TV section called "Play with You" in the "If You Are the One 2" plot, which integrates Beijing's Happy Valley, Mutianyu Great Wall, Tanzhe Temple, Zizhuyuan Park, 798 Art District and other Beijing landmarks into the movie.

\section{Development Strategy of Movie and Television Tourism in Yunnan Based on Industrial Integration Theory}

\subsection{To focus on planning and design, and develop attractive movie and television tourism products.}

Movie and television bases in Yunnan lack comprehensive construction planning and design, and assessment of development trend. As a result, there are so many movie and television bases in Yunnan, but the development was not satisfying. At present, all the movie and television bases in Yunnan are in a state of loss. Because many movie and television bases rely on the support of a 
movie and television work to maintain its operation. Without Chen Kaige's "The Promise", Yuanmou Movie and Television Base will not appear in the public's sight. It restored former loneliness after a brief development. Without continuous presence of movie and television, and perfect movie and television experience, there are fewer movie and television tourists. Therefore, in the establishment of the movie and television base, we must pay attention to planning and design to attract the crew and develop tourism management. We must make a systematic planning. According to the tourists' motivation and psychology in movie and television tourism, we should pay attention to the design of cultural connotation and experiential participatory activities, and enhance the tourism attraction of tourists in the process of developing movie and television tourism products. On one hand, the ideological spirit, cultural awareness and historical elements conveyed by the movie and television are embodied in the products, and are displayed in an appropriate way to meet the needs of tourists to relive their psychology of the classic screen and various cultural experiences. On the other hand, we must pay attention to the development of some participatory and experiential movie and television tourism products, so that tourists can participate in them to improve their enjoyment and enrich the content of tourism activities.

\subsection{To strengthen cooperation with scriptwriters and crews, and innovate movie and television tourism publicity.}

Yunnan is remote in geographical location and its traffic is inconvenient. Many beautiful natural scenery and colorful ethnic customs are not well known to other provinces. The rich movie and television tourism resources are not well known for the whole countries. In this case, it is necessary to expand the publicity channels for movie and television tourism and increase the publicity of Yunnan movie and television tourism, so Yunna may strengthen cooperation with scriptwriters and crews. Various places in Yunnan can make plans to use movie and television works for publicity and marketing according to their own resource characteristics. They can cooperate with scriptwriters, crews and stars, and involve in the movie industry in early site selection, script writing and later movie and television publicity to promote the movie and television base. They can actively use the Internet. At present, in the era of Internet information, people not only inquire about sights and book air tickets for their travel, but even inquire shopping, leisure and entertainment activities in the usual life on the Internet. The network has the characteristics of saving time, flat network and fast response. Movie and television tourism should be good at using the network to carry out various ways of publicity. Drawing on the practices of Hong Kong and the United Kingdom, they can design a movie and television travel map to promote Yunnan tourism. The map vividly and detailedly introduces the places in many movies, including restaurants, streets, shopping malls and attractions. The base can also invite movie stars as the spokesperson for the promotion of Yunnan movie tourism. In this way, designing and creating a brand of a "face-type" product of movie tourism destination in Yunnan will naturally attract the attention of many tourists.

\subsection{To vigorously develop tourism infrastructure of transportation and improve accessibility.}

The transportation industry is an essential element of tourism activities, an important condition for attracting tourists, and a leading factor in the development of the tourism industry. On the whole, Yunnan's transportation industry is still not adapting to the rapid development of tourism, and it is still one of the bottlenecks restricting the development of tourism and movie industry. Therefore, we must continue to promote the construction of tourism transportation infrastructure, such as aviation, railways and highways, especially the construction of railways and highways, to form a safe, comfortable, convenient and fast three-dimensional tourism transportation network to further improve the access conditions of Yunnan tourism and improve the accessibility of tourism destinations.

\subsection{To implement a sustainable development strategy.}

The sustainable development of tourism is an important principle of tourism development, and movie and television tourism is no exception. On one hand, movie and television shooting has a great publicity role for the destination, which can promote local prosperity, and bring positive effect 
to the tourism destination, while can adversely affect the local ecology. For example, after "The Promise" made the stunning alpine azaleas in the Shangri-La Tourmaline Tianchi on the screen, the shooting scene suffers damages which cannot be repaired, so that people can no longer see the beauty of it. Therefore, while attracting movie and television crews, they must also regulate and constrain them, improve and upgrade relevant normative mechanisms, always put the protection of ecological environment and cultural relics first, and resolutely put an end to any form of shooting that damages ecological environment, cultural relics and historic sites. On the other hand, we must also focus on sustainable development in the construction of movie and television bases. We should not blindly spend huge sums of money to build a movie and television base for some short-term popular factors or for a movie whose topic is not easy to repeat. It is necessary to pay attention to long-term development consideration, carry out scientific planning and design, and give a longer life cycle and vitality of the base to achieve sustainable development.

\section{Summary}

Movie and television tourism is not equal to movie + tourism, but is $1+1>2$. Movie and television tourism is the intersection of movie and television culture industry and tourism culture industry. It is a new type of business and profit model. Industrial integration makes movie and television tourism relatively independent. Movie and television tourism has great potential for development. Therefore, following the inherent laws of movie and television tourism, responding flexibly and actively exploring ways will certainly promote the development of the movie and television tourism industry.

\section{Acknowledgments}

Project Fund: Science Research Fund Project of Yunnan Provincial Department of Education (No.2013Y080)

\section{References}

[1] Yin Qingling. A Research on the Countermeasures of the Integration and Development of Movie and Television Tourism Industry [J]. Tourism Overview (The second half of the month), 2017, (10): 149.

[2] Cui Yongjing. A Research on the Development of Yunnan Movie and Television Tourism from the Perspective of Industrial Integration [J]. Journal of Hebei Tourism Vocational College, 2016, 21(01): 8-15.

[3] Zhang Quansheng. A Discussion on the Industrial Mechanism and Development Strategy of Movie and Television Tourism [J]. Qi Lu Realm of Arts, 2014, (02): 105-107.

[4] Wu Jinmei, Song Ziqian. A Research on the Development of Movie and Television Tourism from the Perspective of Industrial Integration [J]. Tourism Tribune, 2011, 26(06): 29-35.

[5] Guo Wen, Huang Zhenfang and Wang Li. A Research on Movie and Television Tourism: A Deep Academic Research -A Literature Review of Domestic and Foreign Movie and Television Tourism in the Past 20 Years [J]. Tourism Tribune, 2010, 25(10): 85-94. 